\title{
Enhancement of Temporally Variable Features in Nailfold Capillary Patterns
}

\author{
P. D. Allen ${ }^{a}$, C. J. Taylor ${ }^{a}$, A. L. Herrick ${ }^{b}$ T.Moore $^{b}$ \\ ${ }^{a}$ Department of Medical Biophysics, Stopford Building, Oxford Road, \\ University of Manchester, Manchester M13 9PT. \\ ${ }^{b}$ University of Manchester, Rheumatic Diseases Centre, \\ Hope Hospital Salford M6 8HD \\ pa@sv1.smb.man.ac.uk
}

\begin{abstract}
Analysis of the condition of blood capillaries in the nailfold is complicated by the fact that gaps in the flow of blood cells results in temporal variability in their appearance. At a single instant the capillaries may be incomplete, but integration of the information from several successive video frames renders the whole capillary visible. This paper shows that combining the information from the video frame sequence by subtracting a multiple of the standard deviation from the mean value for each pixel, improves the signal to noise ratio of variable features when compared with the mean image or using minimum projection.
\end{abstract}

\section{Introduction}

Scleroderma [1] is a connective tissue disease that results in a variety of symptoms stemming from an excessive production of collagen. The most immediately apparent effect is a thickening of the skin causing reduced mobility. Another serious effect is a severe reduction in peripheral circulation exacerbated by exposure to cold, known as Raynaud's phenomenon. Raynaud's phenomenon occurs independently of scleroderma in about 5\% of the population and is nothing more than an irritation that can be dealt with by protecting the extremities from cold. However, for the small number Raynaud's sufferers with scleroderma, the reduction in circulation can become so extreme that amputation of fingers and toes becomes necessary. Improving the peripheral circulation of patients with scleroderma is therefore a major concern to clinicians working in this field. Trials aimed at evaluating possible treatments have been dogged by the relative difficulty of assessing the condition of the peripheral vasculature and quantifying changes brought about by treatments under test.

One technique that has been explored is direct visualisation of the blood capillaries in the skin at the base of the finger nail (nailfold). Here the tiny vessels that link the arterial and venous system can be seen using an optical microscope (see figure 1). In previous work [2], a video camera has been coupled to the microscope, and the condition of the capillary network has been assessed by measuring certain key capillary loop dimensions from single video frames. Gaps in the flow of red blood cells through the capillary loops 
(the capillary wall itself is transparent) mean that not all of the capillary network is visible at any one instant. Thus it would be preferable to integrate the information from a sequence of successive video frames. Also, measuring the capillary dimensions by hand is tedious and therefore error prone.

This work forms part of an on going project to develop a computer based system for the analysis of nailfold capillary patterns. At present, a picture of the complete row of capillary loops nearest the fingernail (distal row) is built up by recording a series of overlapping scenes on video. The proposed system will combine the video frames in each scene to average out temporal variability, and then link those scenes together to form a complete image of the distal row. From this temporally enhanced mosaic the capillary dimensions will be measured automatically. This paper concentrates on finding the best method of integrating the information present in each video sequence.

\section{Video Frame Registration}

Despite being held as still as possible, there will always be some residual motion between finger and microscope during a video sequence. Before the sequence of video frames can be combined, this relative motion must be measured and corrected for. This is complicated by the fact that a single video frame from a nailfold sequence will typically contain several elements besides the actual image of the capillary loops. For example, to make the skin of the nailfold appear more transparent, a layer of oil is applied. Air bubbles can be trapped in this oil and drift across the field of view independently of the finger's relative motion. Light from the lamp illuminating the finger can reflect from the oil resulting in amorphous patches of light which tend to remain stationary in the field of view. Specks of dust in the microscope's optical system also appear stationary, and noise in the video camera/VHS recorder system produces features in the image which have no correlation from one frame to the next. Thus, to register the video frames relative to the capillary network, pixels in each image which belong to the capillaries must be separated from the background. Segmenting curvilinear structures such as blood vessels is a common problem in medical imaging for both 2D [3] and 3D [4] images.

Our solution to this problem has been to apply a linear feature detector used previously to detect line patterns in X-ray mammograms [5]. The result of applying this method to

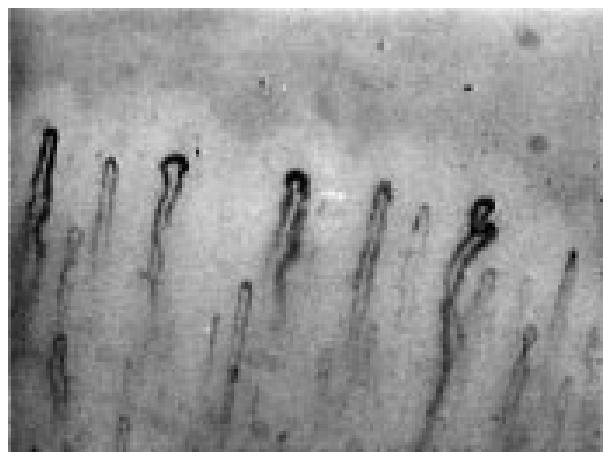

Figure 1: Single video frame showing nailfold capillaries at $\times 200$ magnification.

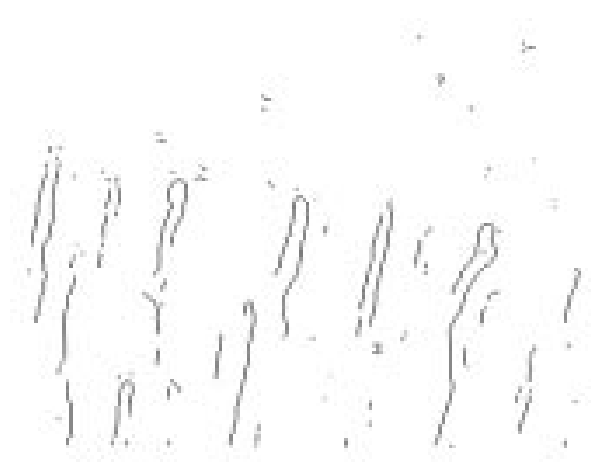

Figure 2: Results of applying linear feature detection to the image in figure 1 . 
the nailfold image in figure 1 is shown in figure 2 . The visible capillaries are reduced to a skeletal representation on pixel thick and it is these remaining pixels which are used to find the transformation from one frame to the next. The extremely narrow depth of field inherent in optical microscopy renders scale changes and perspective distortions due to axial finger motion impossible. Therefore, the transformation from one frame to the next can be described by a combination of rigid translation and rotation. There are a variety of approaches to such an image registration problem [6], and here the translation between two successive frames can be found from the skeletal representations using a Hough transform method. Each of the skeletal pixels from image A are matched with every skeletal pixel from image B. Each match represents a possible translation if rotation is known. If these matches are used to vote in a translation space, a peak emerges at the point representing the translation for which the largest number of pixels in the two images agree. If the two images are miss-aligned by a rotation as well as translation, the size of the peak in the translation image will be smaller than if there had been no rotation. Thus if the translation space is created for a number of possible rotations, the one which yields the highest peak corresponds to the correct, or at least best, rotation. The finger under examination rests in a bracket which constrains rotations to be relatively small.

\section{Combining Images}

Once a sequence of video frames has been registered with respect to the capillary network, the information from the set of images has to be combined in such a way as to make maximum use of the available information. The most obvious approach is to take the mean of the image set, as this averages out the random noise present in the individual images. However, if we consider the case of a capillary in which the column of blood cells contains visible gaps, the image intensity at any point on the capillary will vary with time as the 'lumps' of blood flow through. If an average of the sequence is taken, this variation between light (background) and dark (blood cells) will result in an average value in between and so the difference in intensity between the capillary and the surrounding tissue will be lower than it was in a single frame. Thus, the complete capillary becomes visible, but contrast is lost.

Another commonly used approach is to select the minimum value for each pixel from the sequence. This improves signal contrast, but involves no noise averaging, so it is not obvious that the visibility of the results will be any better than taking the mean.

We propose a third alternative, which is to subtract some multiple of the standard deviation from the mean. The idea is to estimate a value representative of the minimum over the sequence but less susceptible to noise.

To compare the relative merits of the possible approaches, a method of quantifying the resulting contrast is required. If we consider the difference between the intensity of the capillary and the surrounding tissue as the signal, then contrast can be quantified as the signal to noise ratio. More specifically, let us define the mean grey level intensity of red blood cells in a single image as $\bar{S}$ (signal), and the apparent or measured intensity in a composite scene as $S_{m}$. Similarly for the background intensity we have $\bar{B}$ and $B_{m}$. The measured signal to noise ratio in a composite scene can then be written as:

$$
\text { Signal } / \text { Noise }=\frac{B_{m}-S_{m}}{\sqrt{\left(\sigma_{B_{m}}\right)^{2}+\left(\sigma_{S_{m}}\right)^{2}}}
$$


Where $\sigma_{B_{m}}$ and $\sigma_{S_{m}}$ are the noise in $B_{m}$ and $S_{m}$. Using this definition of contrast the three methods of image combination described above can be compared if the four parameters in equation 1 can be defined. To do this the following parameters must be considered:

$\sigma_{S}$ The standard deviation of the intensity of blood cells $\mathrm{S}$, about a mean value $\bar{S}$

$\sigma_{B}$ The standard deviation of the intensity of background B, about a mean value $\bar{B}$

$F_{S}$ The fraction of time for which the value of a pixel belonging to a variable feature corresponds to $\mathrm{S}$.

$N$ The number of samples (In this case the number of images).

As well as $F_{S}$ we would define $F_{B}$, but this is redundant as it is simply $1-F_{S}$. The definition of $F_{S}$ assumes that the variation between blood cells and background is purely bi-modal i.e. there are no values in between. In reality the transition will be slightly less well defined, but since the red blood cells are discrete objects this is a reasonable approximation to work from. The value of $F_{S}$ will not only vary from one capillary to the next, but also within the same capillary. Capillaries can be observed with only a few blood cells spread throughout the whole loop, or alternatively there may be only occasional gaps between streams of cells. Thus the full range of $F_{S}$ from 0 to 1 must be explored. Another assumption that is made in the following is that the noise is not proportional to intensity - in other words $\sigma_{S}$ is equal to $\sigma_{B}$ and both can be replaced by single value $\sigma$. Out of a possible 256 grey levels, the difference between $\bar{S}$ and $\bar{B}$ is only about 20, and so noise would have to increase very rapidly with intensity for any difference to be significant.

\subsection{Image combination by taking the mean}

The background is stable with time and so Gaussian statistics apply. Therefore:

$$
\begin{gathered}
B_{m}=\bar{B} \\
\sigma_{B_{m}}=\frac{\sigma}{\sqrt{N-1}}
\end{gathered}
$$

For a variable feature the distribution of values is the composite of two distributions, those of S and B. The mean of this composite distribution is given by:

$$
\mu=F_{S} \bar{S}+\left(1-F_{S}\right) \bar{B}
$$

Gaussian statistics no longer apply to this distribution and so the uncertainty in the mean cannot be derived from the standard deviation of the composite distribution in the usual way. However, we can assume Gaussian statistics for S and B, and their uncertainties can be combined using error propagation giving:

$$
\sigma_{\mu}=\sqrt{\left(\frac{\sigma F_{S}}{\sqrt{F_{S} N-1}}\right)^{2}+\left(\frac{\sigma\left(1-F_{S}\right)}{\sqrt{\left(1-F_{S} N\right)-1}}\right)^{2}}
$$

For a scene in which the mean of the image sequence is taken, $S_{m}=\mu$, and $\sigma_{S_{m}}=\sigma_{\mu}$. 


\subsection{Image combination using minimum projection}

The extreme value (in this case the minimum) of a Gaussian distribution is in itself a random variable and has a probability distribution with expectation value and standard deviation given by [7]:

$$
\begin{gathered}
E\left(X_{\min }\right)=\mu-\sigma\left(\xi+\frac{0.5772}{\xi}\right) \\
\sigma_{\min }=\sigma \frac{\pi}{\sqrt{6}} \frac{1}{\xi}
\end{gathered}
$$

where $\xi \approx \sqrt{2 \ln N-\ln (4 \pi \ln N)}, N$ is the size of sample from which the minimum was drawn, and $\mu$ is the mean of the underlying distribution.

For the background, the value of $B_{m}$ is given by equation 3 by replacing $\mu$ with $\bar{B}$, and $\sigma_{B_{m}}$ is given by equation 4 as it stands.

For a variable capillary, if $\sigma$ is small compared with $(\bar{S}-\bar{B})$ (which is true for the nailfold images used in this study) then the minimum values can be assumed to have been drawn from the distribution of $\mathrm{S}$ values only i.e. the values of $S_{m}$ and $\sigma_{S_{m}}$ are given by replacing $\mu$ with $\bar{S}$ and $N$ with $F_{S} N$ in equations 3 and 4 .

\subsection{Image combination using $\mu-n \sigma$}

For the background we can apply Gaussian statistics. The mean $\mu$ has an uncertainty of $\frac{\sigma}{\sqrt{N-1}}$ and the standard deviation $\sigma$ an uncertainty of $\frac{\sigma}{\sqrt{2(N-1)}}$. If $B_{m}$ is derived from $\mu-n \sigma$ where $n$ is some constant, then error propagation yields:

$$
\sigma_{B_{m}}=\sigma \sqrt{\frac{2+n^{2}}{2(N-1)}}
$$

Adding the errors in quadrature assumes that the uncertainties in $\mu$ and $\sigma$ are uncorrelated. This seems at first dubious since the definition of $\sigma$ includes $\mu$. However, using a simulation based on numbers drawn randomly from a Gaussian distribution, it is relatively straight forward to verify equation 5. For example: Generating a Gaussian distribution of $\mu=100, \sigma=10$, and number of samples $N=100,10000$ times and calculating $\mu-3 \sigma$ for each iteration gives a standard deviation $\sigma_{\mu-3 \sigma}$ of $2.34 \pm 0.02$. Equation 5 gives $\sigma_{B_{m}}=2.36$ for the same parameters.

As mentioned above, the distribution of intensity values of a pixel belonging to a variable feature is a composite of the two distributions corresponding to $\mathrm{S}$ and $\mathrm{B}$. The mean of this composite function is given by equation 2. To calculate the value of $\mu-$ $n \sigma$, the standard deviation of the composite function $\sigma_{c}$ must be calculated. Using the following definition of standard deviation: $\sigma^{2}=E\left(x^{2}\right)-\bar{x}^{2}$, the standard deviation of a composite function can be shown to be:

$$
\sigma_{c}^{2}=F_{S}\left(\sigma_{S}^{2}+\bar{S}^{2}\right)+\left(1-F_{S}\right)\left(\sigma_{B}^{2}+\bar{B}^{2}\right)-\mu^{2}
$$

Where $\mu$ is given by equation 2 . Again it is relatively simple to verify this expression with simulation. 
The value of $S_{m}$ in this case is given by $\mu-n \sigma_{c}$. An expression for $\sigma_{S_{m}}$ can be found using error propagation. The variables that determine $S_{m}$ are $F_{S}, n, \bar{S}, \bar{B}, \sigma_{S}$ and $\sigma_{B}$. Here, the distinction between $\sigma_{S}$ and $\sigma_{B}$ is made because, though their values are assumed to be the same, the uncertainties on their estimates are defined as $\frac{\sigma}{\sqrt{F_{S} N-1}}$ and $\frac{\sigma}{\sqrt{\left(1-F_{S}\right) N-1}}$ respectively. $F_{S}$ and $n$ are assumed constant in any particular example and so $\sigma_{S_{m}}$ can be defined in the following way:

$$
\sigma_{S_{m}}=\sqrt{\left(\sigma_{S} \frac{\delta S_{m}}{\delta S}\right)^{2}+\left(\sigma_{B} \frac{\delta S_{m}}{\delta B}\right)^{2}+\left(\sigma_{\sigma_{S}} \frac{\delta S_{m}}{\delta \sigma_{S}}\right)^{2}+\left(\sigma_{\sigma_{B}} \frac{\delta S_{m}}{\delta \sigma_{B}}\right)^{2}}
$$

The partial differentials can be shown to be:

$$
\begin{aligned}
\frac{\delta S_{m}}{\delta \bar{S}} & =\frac{F_{S}-\frac{n}{2}}{\alpha\left(2 F_{S}(\bar{S}-\bar{B})+2 F_{S}^{2}(\bar{B}-\bar{S})\right)} \\
\frac{\delta S_{m}}{\delta \bar{B}} & =\frac{\left(1-F_{S}\right)-\frac{n}{2}}{\alpha\left(2 F_{S}(\bar{B}-\bar{S})+2 F_{S}^{2}(\bar{S}-\bar{B})\right)} \\
\frac{\delta S_{m}}{\delta \sigma_{S}} & =\frac{-n}{\alpha F_{S} \sigma_{S}} \\
\frac{\delta S_{m}}{\delta \sigma_{B}} & =\frac{-n}{4 \alpha\left(1-F_{S}\right) \sigma_{B}}
\end{aligned}
$$

Where $\alpha=\sqrt{F_{S}\left(\sigma_{s}^{2}+\bar{S}^{2}-\sigma_{B}^{2}+\bar{B}^{2}-2 \bar{S} \bar{B}\right)+F_{S}^{2}\left(2 \bar{S} \bar{B}-\bar{B}^{2}-\bar{S}^{2}\right)+\sigma_{B}^{2}}$

\subsection{Comparison of image combination techniques}

With the equations given above, we are in a position to compare the signal to noise ratio as a function of $F_{S}$ for the three techniques; mean, minimum projection and $\mu-n \sigma$. Figures 3 and 4 show the signal to noise ratio as a function of $F_{S}$ for a sample number of 100. In figure 3, $\sigma$ is a tenth of the difference between capillary and background $(\bar{B}-\bar{S})$. For most values of $F_{S}, \mu-\sigma$ results in the best signal to noise ratio, $\mu-3 \sigma$ is a slight improvement below $F_{S}=0.3$, and the mean gives the best results above $F_{S}=0.85$. Minimum projection is only shown here for $F_{S}>0.1$, below this value the equations used to calculate the signal to noise ratio break down since the number of samples of $\mathrm{S}$ (i.e. $F_{S} N$ ), is too small for the assumptions involved in the derivation to hold.

Figure 4 shows results for a much smaller signal to noise ratio obtained by making $\sigma$ three times $(\bar{B}-\bar{S})$, here it is clear that taking the mean produces the best signal to noise ratio for any degree of feature variability. Below $F_{S}=0.4$, minimum projection results in zero signal to noise ratio. This is because the minimum expectation value given by equation 3 is a function of $\mathrm{N}$. Throughout the range of $F_{S}$, the number of samples of background B are the same, however, the number of samples of S are decreasing and so the expectation value of $S_{\min }\left(S_{m}\right.$ in the nomenclature of the previous derivations) is also decreasing. For low enough values of $F_{S}-0.4$ in this example - $S_{m}$ can become lower than $B_{m}$, in which case the process of minimum projection would select $B_{m i n}$ rather than $S_{\text {min }}$ and so $\left(B_{m}-S_{m}\right)=\left(B_{m}-B_{m}\right)=$ zero. 


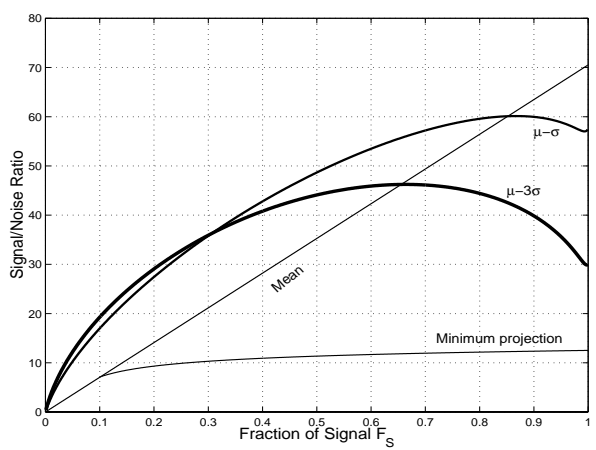

Figure 3: Signal to noise ration as a function of feature variability $F_{S} \cdot \bar{B}-\bar{S}=1$, $\sigma=0.1, N=100$.

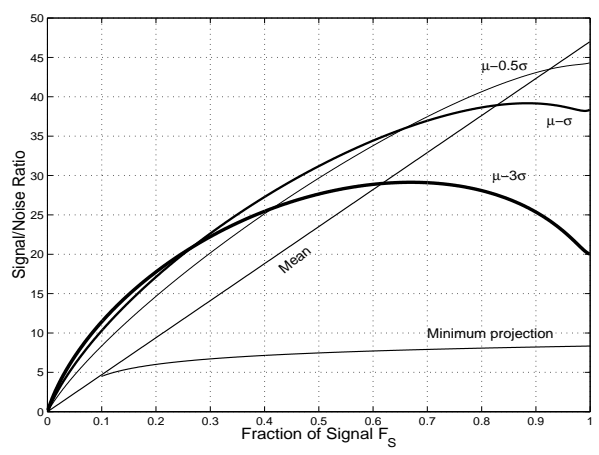

Figure 5: Signal to noise ratio as a function of feature variability $F_{S} . \bar{B}-\bar{S}=20$, $\sigma=3, N=100$.

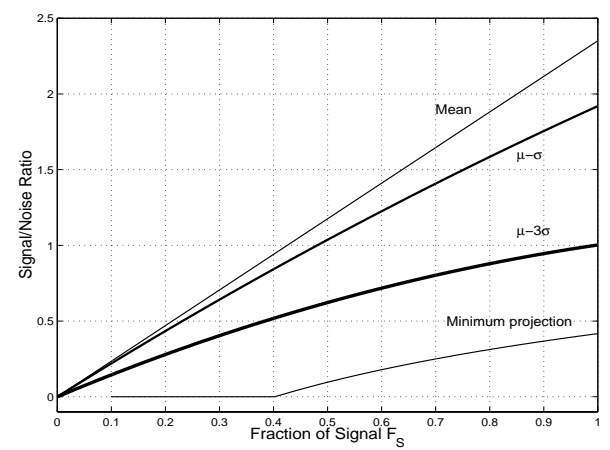

Figure 4: Signal to noise ration as a function of feature variability $F_{S} . \bar{B}-\bar{S}=1$, $\sigma=3, N=100$.

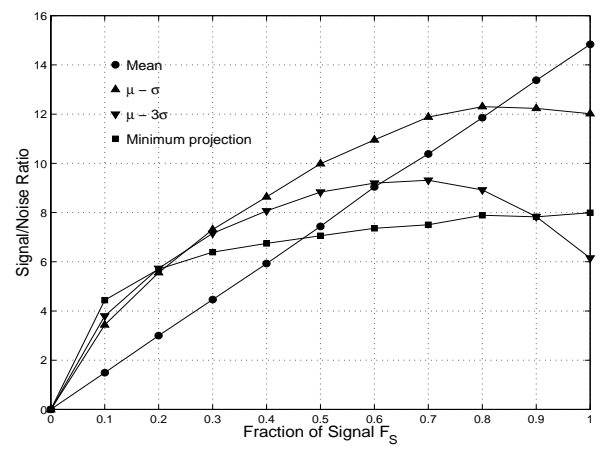

Figure 6: Signal to noise ration as a function of feature variability $F_{S} . \bar{B}-\bar{S}=20$, $\sigma=3, N=10$ (Simulation).

Determining the best method of image combination clearly depends on estimating the appropriate values for $(\bar{B}-\bar{S})$, and $\sigma$. For the nailfold images used in this study, $(\bar{B}-\bar{S})$ and $\sigma$ are approximately 20 and 3 respectively. The results of using these figures for a sample size $N$ of 100 is shown in figure 5. As in figure 3, the optimum method of image combination is a function of $F_{S}$, but for most values of $F_{S}, \mu-\sigma$ gives the best results.

It remains to explore the dependency on sample size $N$. For the data acquisition and analysis scheme under development, 100 images per scene may be unfeasible within reasonable time and memory constraints and so it is important to know what effects smaller sample sizes will have.

For a sample size of 10 , the equations used to predict the signal to noise ratio for minimum intensity projection become unreliable. Also, for $\mu-n \sigma$, if $N=10$, then when $F_{S}$ is 0.1 there is only one sample from $\mathrm{S}$, and for $F_{S}=0.9$ there is only one sample of B. For one sample the statistical arguments upon which the equations for $S_{m}$ and $B_{m}$ are based become meaningless and the $(N-1)$ 's result in asymptotic behaviour. To obtain a reliable estimate of the signal to noise ratio for $N=10$ we can instead use 
numerical simulations.

Figure, 6 shows the result of simulating minimum projection and $\mu-n \sigma$ for $\mathrm{N}=10$. These simulations are the result of 100000 trials, and the errors at each point are approximately the size of the markers used. Closer inspection of the equations used to calculate the signal to noise ratio for $\mu-n \sigma$ reveal an approximate proportionality to $\sqrt{N}$, thus we would expect the signal to noise ratio for $\mu-n \sigma$ to be approximately a factor of $\sqrt{10}$ greater in figure 5 than in figure 6 , which is the case, providing a useful verification of the mathematics in the previous sections. The signal to Noise ratio resulting from minimum projection has a different relationship to $N$, and it can be seen from figure 6 that at $N=10$ results comparable to $\mu-3 \sigma$ are achieved. In particular, for $F_{S}=0.1$, minimum projection actually produces the the best signal to noise ratio. However, $\mu-\sigma$ again provides the best results for most values of $F_{S}$.

\section{Applying Image Combination Techniques to Data}

The results of applying the image combination techniques described in section 3 to a sequence of 20 nailfold images at $\times 200$ are shown in figures 7 to 12 . The appearance of the central capillary loop was variable with time and in the single video frame shown in figure 7, gaps in the loop can be seen. Figure 8 shows the standard deviation for each pixel in the image after the sequence had been registered with respect to the capillary loops. As expected the central loop stands out as being the most variable feature. Relatively high variability is also apparent around the edge of some of the other capillary loops - most notably that of the capillary in the left hand corner. This can be explained by the variation in the appearance of the edge of the capillary as the blood cells pass through - an effect

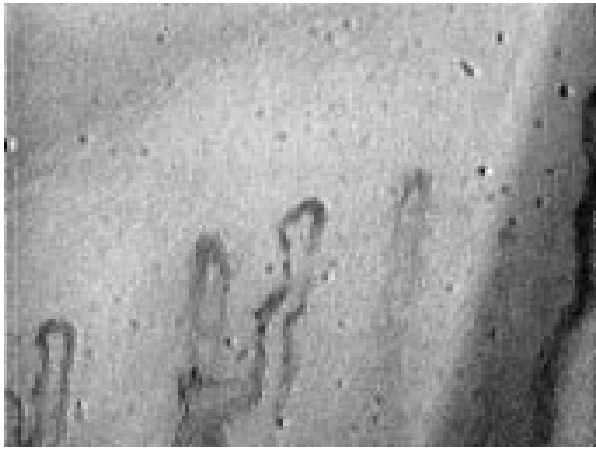

Figure 7: Single video frame showing nailfold capillaries at $\times 600$ magnification.

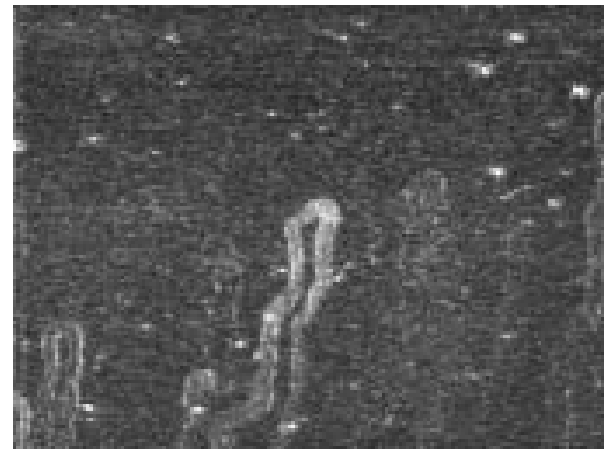

Figure 8: The standard deviation for a registered sequence of 20 video frames.

observable in the video sequence. However, it would also be consistent with a missregistration of the sequence, and so further study into the accuracy of the registration technique may be necessary.

Figure 9 shows the result of taking the mean of the image sequence. The central capillary loop now appears complete and the noise in the scene has been reduced. The capillary loops either side of the central loop still appear relatively indistinct - this is because they are slightly deeper in the skin than the others. With the reduction in noise, 


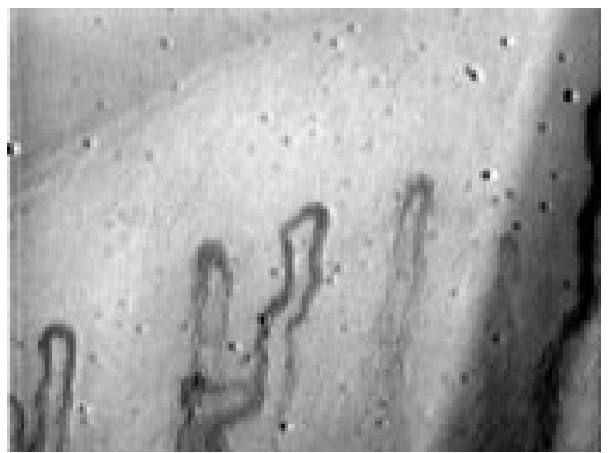

Figure 9: The mean pixel values of the registered sequence.

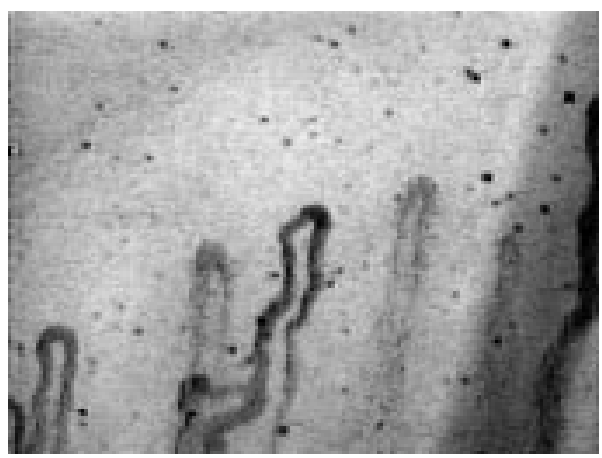

Figure 11: The mean image minus three standard deviations.

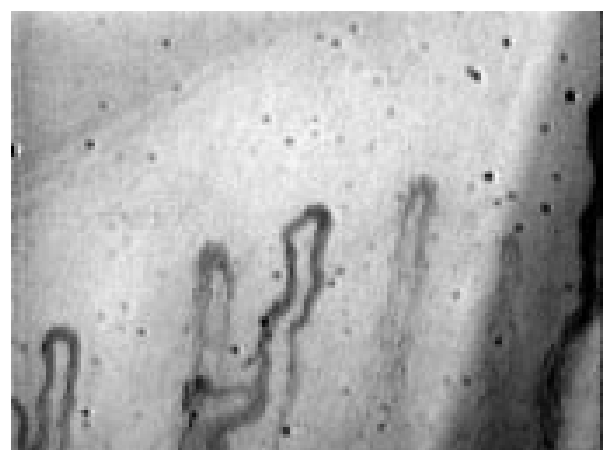

Figure 10: The mean image minus one standard deviation.

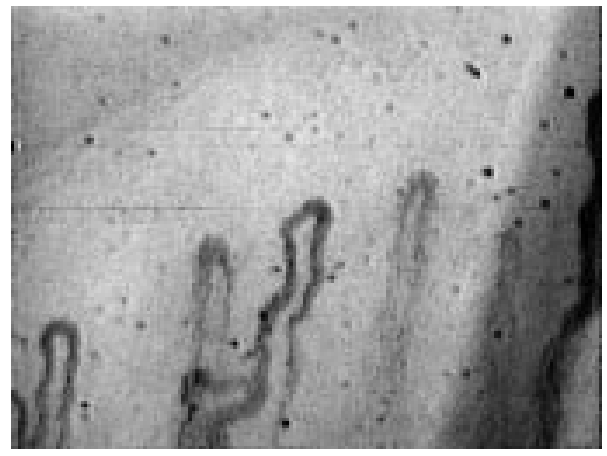

Figure 12: The minimum pixel values from the image sequence.

the texture of the skin obscuring them is just becoming apparent. Figure 10 shows the result of subtracting one standard deviation from the mean image. The contrast of the central capillary has been enhanced but there has been a slight increase in the noise levels of the surrounding temporally stable background. In figure 11, three standard deviations have been subtracted from the mean. The central capillary loop has been greatly darkened, but the noise overall noise levels are comparable to that of a single frame. Using minimum projection (figure 12) has a similar effect to $\mu-3 \sigma$, but the contrast of the central capillary is slightly lower. Overall the $\mu-\sigma$ in figure 10 is the clearest, supporting our theoretical predictions.

\section{Conclusions}

The signal to noise ratio estimates in figure 5 show that for the noise and contrast levels found in nailfold images, the best signal to noise ratio is achieved by using $\mu-n \sigma$, where the value of $n$ is a function of the degree of temporal variability $F_{S}$. If the contrast between blood cells and surrounding tissue $(\bar{B}-\bar{S})$ and the noise level $\sigma$ is known and can 
be assumed to be constant, then the value of $F_{S}$ can be derived at each pixel position from equation 2. However, the relative values of $\bar{S}$ and $\bar{B}$ vary from one patient to the next. The extent of this variation and whether this will render evaluation of $F_{S}$ impracticable requires further study. However, figure 5 suggests that subtracting between a half and one standard deviation gives an improved signal to noise ratio over the mean for most values of $F_{S}$. Also the set of images in section 4 show that using $\mu-\sigma$ improves the contrast of the variable capillary without noticeable degradation of stable ones. Thus, in the absence of a reliable estimate of $F_{S}, \mu-\sigma$ would seem to be the best option for image combination of the nailfold images encountered in this study.

The signal to noise ratio predictions shown in figure 5 are specific to the data collected for this study, but the mathematical framework described in section 3 is applicable to any problem requiring temporal or spatially varying data to be compounded. The specificity of figure 5 is in the assumed ratio between signal and noise in an individual frame, or $(\bar{B}-\bar{S})$ vs $\sigma$. For simplicity we have also assumed that the noise in an image is independent of signal. This is a valid assumption for the nailfold images, but the derivations of signal to noise ratio do not depend on this and so a known relationship between signal and noise can be accommodated in the framework described.

\section{Acknowledgments}

This project is funded by The Raynaud's and Scleroderma Association, 112 Crewe Road, Alsagar, Cheshire ST7 2JA (Charity Reg. No. 326306)

\section{References}

[1] D. A. Isenberg and C. Black. Raynaud's Phenomemon, Scleroderma, and Overlap Syndromes. British Medical Journal, 310:795-798, March 1995.

[2] M. Bukhari, A.L. Herrick, T. Moore, J. Manning, and M. I. V. Jayson. Increased Nailfold Capillary Dimensions in Primary Raynaud's Phenomenon and Systemic Sclerosis. British Journal of Rheumatology, 35:1127-1131, 1996.

[3] R. Kutka and S. Stier. Extraction of Line Properties Based on Direction Fields. IEEE Trans. Med. Imag, 15(1):51-58, February 1996.

[4] Y. Sato, S. Nakajima, N. Shiraga, H. Atsumi, S. Yoshida, T. Koller, G. Gerig, and R. Kikinis. Three-Dimensional Multi-Scale Line Filter for Segmentation and Visualization of Curvilinear Structures in Medical Images. IEEE Trans. Med. Imag, 2(2):143-168, June 1998.

[5] R. Zwiggelar, T. Parr, and C. Taylor. Finding Oriented Line Patterns in Digital Mammographic Images. In Proc. of 7th BMVC Edinburgh, 1996.

[6] L. G. Brown. A Survey of Image Registration Techniques. ACM Computing Surveys, 24(4):321-376, December 1992.

[7] Extreme Value Distributions. http://www.dnv.no/ocean/bk/c/a43/s0.htm. 